\title{
Erratum to: MRI characterization of temporal lobe epilepsy using rapidly measurable spatial indices with hemisphere asymmetries and gender features
}

\author{
Siddhartha Datta ${ }^{1} \cdot$ Sudipta Sarkar $^{2} \cdot$ Sumit Chakraborty $^{2} \cdot$ Sai Krishna Mulpuru ${ }^{3}$. \\ Swadhapriya Basu ${ }^{2}$ - Basant K. Tiwary ${ }^{4}$. Nilkanta Chakrabarti ${ }^{1}$. \\ Prasun Kumar Roy ${ }^{5,6}$
}

Published online: 19 July 2015

(C) Springer-Verlag Berlin Heidelberg 2015

Erratum to : Neuroradiology

DOI 10.1007/s00234-015-1540-6

The initial publication of Table 2 contained errors due to author error during the proofing stage. The corrected version of Table 2 is below. The data marked in bold were misplaced in the original publication.

The online version of the original article can be found at http://dx.doi.org/ $10.1007 / \mathrm{s} 00234-015-1540-6$.

\section{Nilkanta Chakrabarti}

ncphysiolcu@gmail.com

1 Department of Physiology and UGC-CPEPA Centre for "Electro-physiological and Neuro-imaging studies including Mathematical Modelling", University of Calcutta, 92 APC Road, Kolkata 700009, India

2 Department of Radiodiagnosis, IPGME\&R, SSKM Hospital, Kolkata, India

3 National Neuro-Imaging Facility, National Brain Research Centre, Manesar, India

4 Centre for Bioinformatics, School of Life Sciences, Pondicherry University, Pondicherry, India

5 Computational Neuroimaging Division, National Brain Research Centre, Manesar, India

6 Clinical Neuroscience Unit, National Brain Research Centre, General Hospital Campus, Gurgaon, India 


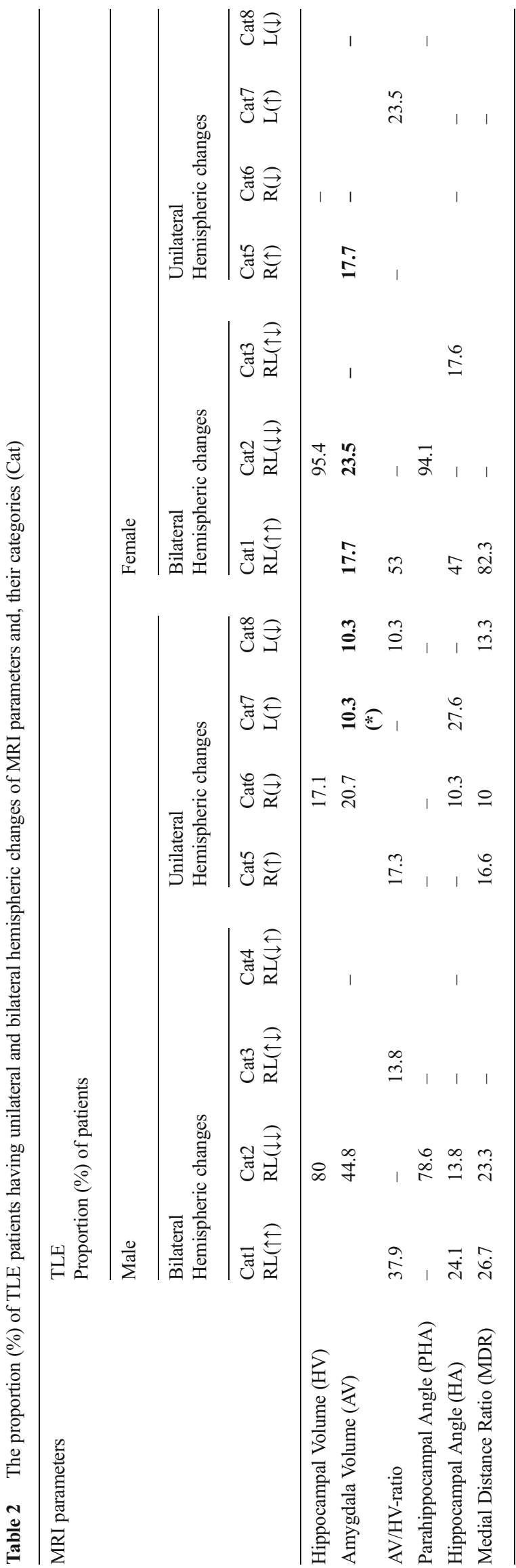

